\title{
Non Obese and Obese Women with Polycystic Ovary Syndrome- Their Metabolic Characteristics and Insulin Resistance
}

\author{
Dr. Pradnya G. Dongargaonkar ${ }^{1}$, Dr. Gautam S. Aher ${ }^{2^{*}}$, Dr. Urmila G. Gavali ${ }^{3}$ \\ ${ }^{1}$ Resident, Professor and Head of Department, ${ }^{2}$ Associate Professor, ${ }^{3}$ Department of Obstetrics and Gynaecology, Dr. Vithalrao Vikhe Patil Medical \\ College and Hospital, Ahmednagar-414111 Maharashtra, India
}

DOI: $10.36348 /$ sijog.2020.v03i03.002 $\quad$ | Received: 19.02 .2020 | Accepted: 26.02 .2020 | Published: 18.03 .2020

*Corresponding author: Dr. Gautam S. Aher

Abstract

Polycystic ovary syndrome (PCOS) is found in 6-10\% of the general population and considered to be a common hormonal disorder in females of reproductive age. It is associated with multiple factors genetic and environmental and has varied morphological and metabolic presentations. Even though insulin resistance is seen in these patients, some are obese while some PCOS patients are lean. This implies a difference in the metabolic characteristics of these patients. Thus, this study was carried out to study the metabolic characteristics and the Insulin resistance.

Keywords: Polycystic Ovarian Syndrome, Insulin resistance, Metabolism

Copyright @ 2020: This is an open-access article distributed under the terms of the Creative Commons Attribution license which permits unrestricted use, distribution, and reproduction in any medium for non-commercial use (NonCommercial, or CC-BY-NC) provided the original author and source are credited.

\section{INTRODUCTION}

Polycystic ovarian syndrome is one of the most common endocrine disorder in women of reproductive age with prevalence ranging from $2.2 \%$ to $26 \%$ worldwide [1]. According to the revised Rotterdam PCOS diagnostic criteria any 2 of the following should be presentoligoovulation/anovulation, clinical or biochemical signs of hyperandrogenism, appearance of polycystic ovaries on ultrasound [2]. Classically the disorder is lifelong, characterized by abnormal menses from puberty, to acne and hirsutism arising in teens, infertility, emergence of obesity early in adulthood presumably accompanied by increasing hyperinsulinaemia, dyslipidemia, and increased incidence of cardiovascular complication later in life[3]. There are only few studies concerning the correlations between phenotypic expression, body composition and PCOS, and relationship with the processes of growth and sexual maturation and metabolic changes. Also not all patients with PCOS are obese. However irrespective of presence of other risk factors patients with PCOS have been reported to have metabolic abnormalities, insulin resistance, dyslipidaemia and complication later in life. So this study was conducted to study the metabolic characteristics of patients with PCOS- in both obese (BMI $>30 \mathrm{~kg} / \mathrm{m} 2)$ and non obese women $(\mathrm{BMI}<30 \mathrm{~kg} / \mathrm{m} 2)$.

Material and method: It was a cross sectional study conducted from August 2018 to March 2019, at a tertiary care centre in western Maharashtra. A total of 100 women with PCOS were taken in the study (oligomenorrhoea, sonological appearance of PCOS, biochemical or clinical hyperandrogenism) 50 of these were obese- BMI $>25 \mathrm{~kg} / \mathrm{m} 2$ and 50 non obese womenBMI $<25 \mathrm{~kg} / \mathrm{m} 2.50$ controls were taken in each group with similar BMI. All women underwent through history taking, physical examination, and anthropometric assessment (height, weight, BMI and waist hip ratio). Patients were specifically asked for irregular menstrual cycles in the form of oligomenorrhoea, hirsutism, infertility, family history (diabetes/PCOS/Hypertension), dietary intake and data regarding their physical activity was noted as per the global Physical activity questionnaire. Signs of insulin resistance such as acanthosis nigrans and skin tags were noted. All women underwent several biochemical testsfasting blood glucose, $2 \mathrm{hr}$ post $75 \mathrm{gm}$ blood glucose, $\mathrm{HbA} 1 \mathrm{C}$, fasting lipid profile, fasting insulin and serum testosterone. All women also underwent biochemical tests for ruling out secondary causes of PCOS (prolactin, thyroid stimulating hormone, serum 
Pradnya G. Dongargaonkar et al; Sch Int J Obstet Gynec, March. 2020; 3(3): $72-74$

cortisol). Insulin resistance was measured by increased fasting insulin levels $>12.2 \mu \mathrm{U} / \mathrm{ml}$. An elevated wait to hip ratio was considered as the clinical marker for visceral adiposity.

\section{RESULTS}

Of the total patients with PCOS $21 \%$ were diagnosed with metabolic syndrome (International
Diabetic Federation criteria), 33\% women had impaired glucose tolerance and 5\% women had Diabetes mellitus. Even amongst the obese patients the wait to hip ration was significantly more in patients with PCOS patients signifying a tendency for visceral adiposity as against subcutaneous adiposity.

\begin{tabular}{|l|l|l|l|l|}
\hline \multicolumn{5}{|c|}{ Baseline characteristics of PCOS patients (obese and Non-obese) and the controls } \\
\hline \multirow{3}{*}{ Variables } & Obese group & Non obese group & $\begin{array}{l}\text { Controls } \\
\mathrm{n}=25\end{array}$ \\
\cline { 2 - 5 } & $\begin{array}{l}\text { PCOS } \\
\mathrm{n}=25\end{array}$ & $\begin{array}{l}\text { Controls } \\
\mathrm{n}=25\end{array}$ & $\begin{array}{l}\text { PCOS } \\
\mathrm{n}=25\end{array}$ & 25 \\
\hline Age $(\mathrm{yrs})$ & 23.16 & 23.16 & 154 & 24 \\
\hline Height $(\mathrm{cm})$ & 153 & 155 & 53 & 51 \\
\hline Weight $(\mathrm{kg})$ & 74 & 72 & 22 & 21 \\
\hline BMI $\left(\mathrm{kg} / \mathrm{m}^{2}\right)$ & 30 & 30 & 82 & 72 \\
\hline Waist $(\mathrm{cm})$ & 95 & 91 & 93 & 86 \\
\hline Hip $(\mathrm{cm})$ & 103 & 104 & 0.9 & 0.8 \\
\hline Waist-hip ratio & 0.9 & 0.8 & & \\
\hline
\end{tabular}

\begin{tabular}{|l|l|l|l|l|}
\hline \multicolumn{5}{|c|}{$\begin{array}{c}\text { Comparison of metabolic parameters, insulin resistance indices, and body composition parameters } \\
\text { between polycystic ovary syndrome women (obese and non obese) and controls (obese and non obese) }\end{array}$} \\
\hline \multirow{2}{*}{ Variable } & Obese group & \multicolumn{2}{l|}{ Non obese group } \\
\cline { 2 - 5 } & PCOS & Control & PCOS & Control \\
\hline Serum testosterone (ng/dL) & 40 & 34 & 32 & 31 \\
\hline Fasting glucose (mg/dL) & 97 & 90 & 94 & 85 \\
\hline Blood glucose post prandial (mg/dL) & 121 & 111 & 103 & 104 \\
\hline HbA1c $(\%)$ & 5.6 & 5.5 & 5.4 & 5.2 \\
\hline Total cholesterol $(\mathrm{mg} / \mathrm{dL})$ & 173 & 157 & 175 & 147 \\
\hline Triglycerides $(\mathrm{mg} / \mathrm{dL})$ & 88 & 92 & 80 & 73 \\
\hline HDL-cholesterol $(>60 \mathrm{mg} / \mathrm{dL})$ & 44 & 40 & 50 & 42 \\
\hline LDL--cholesterol $(<100 \mathrm{mg} / \mathrm{dL})$ & 109 & 103 & 107 & 93 \\
\hline Fasting Insulin $(0-30 \mu \mathrm{U} / \mathrm{ml})$ & 26 & 10 & 10 & 6.4 \\
\hline
\end{tabular}

\section{RESULTS}

A total of 100 PCOS women (obese- 50 non obese 50) and 100 controls were included in the study. All women had decreased frequency of cycles, hyperandrogenism- biochemical or clinical or sonological appearance of polycystic ovaries. Demographic and baseline characteristics of obese PCOS patients and controls, and non-obese PCOS patients and controls are given in table 1 . We observed that for a given BMI and weight of the patient, the waist circumference between obese PCOS and control group was statistically significant with waist circumference in obese PCOS patients being higher than that their control counterparts. This means other than obesity, waist circumference can also be used as an individual parameter for assessment of metabolic abnormality in obese patients with PCOS. Comparison of metabolic parameters, insulin resistance indices, and body composition parameters between polycystic ovary syndrome women (obese and non obese) and controls (obese and non obese) is given in table-2. We observed that the plasma blood sugar level in patient's non obese patients with PCOS was normal. However, obese patients with PCOS had deranged BSL- fasting and post prandial values as compared to obese patients without PCOS, with a statistically significant difference $(\mathrm{P}<$ $0.001)$. The testosterone value in the 50 obese patients with PCOS was $40 \pm 2.398$, against those obese without PCOS who had average testosterone level of $34 \pm 107$ which is statistically significant, implying that there is significant hyperandrogenism in patient with PCOS who are also obese. The serum testosterone values in non-obese patients with PCOS were $32 \pm 2.145$ and that without PCOS was $31 \pm 1.983$ which is not statistically significant. The serum fasting insulin level in obese patients with PCOS was $26 \pm 1.342$, while that in obese without PCOS was $10 \pm 4.134$, which is statistically significant. The serum fasting insulin levels in non obese patients with PCOS was $10 \pm 1.951$ while that in the non obese controls was $6.4 \pm 2.743$ which is again statistically significant, which implies that PCOS was associated with insulin resistance in both obese and non obese patients. 


\section{DISCUSSION}

The study was conducted with an attempt to understand the body composition, metabolic characteristics in age and BMI comparable patients, with and without PCOS. The literature available for this with respect to Indian population is limited.

In our study we found that there is significant difference in the waist circumference of the PCOS patients as compared to their controls in both obese and non-obese patients. This means that waist circumference can also be used as a parameter for risk assessment for PCOS. In study conducted by Pazderska A et al. in 2018, the univariate correlations and stepwise regression modelling identified waist circumference (WC), as a better surrogate than PCOS status, independently predicting multiple variables of glucose and lipid metabolism [4]. Body-mass index (BMI) and waist circumference (WC) are surrogate markers of obesity, especially visceral adiposity [5]. WC is a common indicator of central obesity, thus it is facilitated in detecting MetS[6].

In our study all the patients with PCOS irrespective of obesity had insulin resistance as compared to their respective controls. There was significant rise in the serum fasting insulin level in patients with PCOS as compared to their controls, indicating that patients with PCOS are metabolically worse than their age and BMI comparable counterparts. Various genetic and environmental factors might further be contributory. Overall, insulin resistance and the compensatory hyperinsulinemia affects some 65$70 \%$ of women with PCOS [7, 8]. In fact study conducted by John Marshall in 2012 recommends treatment of all patients of PCOS for insulin resistance given that, use of Metformin for same has both metabolic and reproductive advantages [9].

Even though patients with PCOS had increased tendency for insulin resistance, the non obese patients with PCOS had normal blood sugar levels. However, obese PCOS patients had tendency for deranged blood sugar levels. With a four-fold increased risk of type 2 diabetes (DM2), the Population Attributable Risk of DM2 that could be avoided if PCOS were eliminated is a substantial 19-28\% of women of reproductive age [10]. Since the patients with PCOS are of reproductive age group they theoretically carry an increased risk for obstetric morbidities such as gestational diabetes mellitus and its associated complications.

\section{CONCLUSION}

Patients with PCOS have an increased tendency for visceral and peripheral adiposity with increased propensity for metabolic complications in the long run, with earlier age of onset of same as compared to their BMI and age controlled controls.

\section{REFERENCES}

1. Body composition and polycystic ovary syndrome. (2010). Zabuliene L, Tutkuviene J. Medicina (Kaunas); 46 (2): 142-157.

2. Rotterdam ESHRE/ASRM -Sponsored PCOS consensus Workshop Group.(2004). Revised 2003 consensus on diagnostic criteria and long term health risks related to polycystic ovary syndrome. Fertil Steril, 81:19-25.

3. Peserico, A., Angeloni, G., Bertoli. (1989). Prevelance of polycystic ovaries in women with acne. Arch Dermatol Res, 281:502-503.

4. Pazderska, A., Kyaw, T.T., Phelan, N., McGowan, A., Sherlock, M., Behan, L., Boran G., Gibney, J.(2018). In women with PCOS, waist circumference is a better surrogate of glucose and lipid metabolism than disease status per se. Clin Endocrinol (Oxf), 88(4):565-574.

5. Li, R., Zhang, Q., Yang, D. (2013). Prevalence of polycystic ovary syndrome in women in China. A large community based study. Hum Reprod, 28: 2562-9.

6. Zhu, S., Wang, Z., Heshka, S.(2002). Waist circumference and obesity associated risk factors among whites in the third National Health and Nutrition Examination Survey clinical action thresholds. Am J Clin Nutr, 76: 743-9.

7. Dunaif, A., Segal, K.R., Futterweit, W., Dobrjansky, A. (1989). Profound peripheral insulin resistance, independent of obesity, in polycystic ovary syndrome. Diabetes, 38:1165-74.

8. DeFronzo, R.A., Tobin, J.D., Andres, R.(1979). Glucose clamp technique: a method for quantifying insulin secretion and resistance. Am J Physiol, 237:E214-E23.

9. Marshall, J.C., Dunaif, A.(2012). Should all women with PCOS be treated for insulin resistance?. Fertil Steril, 97(1):18-22.

10. Rodgers, R.J., Avery, J.C., Moore, V.M. (2019). Complex diseases and co-morbidities: polycystic ovary syndrome and type 2 diabetes mellitus. Endocr Connect, 8(3):R71-R75. 